Einführung zum Thema

Gastroenterologe 2019 $14: 413-414$

https://doi.org/10.1007/s11377-019-00385-y

(c) Springer Medizin Verlag GmbH, ein Teil von Springer Nature 2019

\section{B. Siegmund' $\cdot$ G. Rogler ${ }^{2}$}

'Medizinische Klinik mit Schwerpunkt Gastroenterologie, Infektiologie, Rheumatologie, Charité Universitätsmedizin Berlin, Campus Benjamin Franklin, Berlin, Deutschland

${ }^{2}$ Klinik für Gastroenterologie und Hepatologie, UniversitätsSpital Zürich, Zürich, Schweiz

\title{
Eine neue Dynamik in den Therapiestrategien der chronisch-entzündlichen Darmerkrankungen
}

Die Entwicklung der Therapiestrategien bei chronisch-entzündlichen Darmerkrankungen (CED) befindet sich in einer Phase zunehmender Dynamik. Die sich damit erhöhende Komplexität der Therapie stellt für uns als behandelnde Ärztinnen und Ärzte eine neue Herausforderung dar. Gleichzeitig bedeutet dies für unsere Patientinnen und Patienten neue Möglichkeiten und damit unter Umständen auch eine bessere Lebensqualität. Mit der vorliegenden Ausgabe möchten wir auf die aktuellen Therapiealgorithmen für CED eingehen und hier neben den therapeutischen Innovationen durchaus auch die Stärke der „konservativen“ Medikamente hervorheben. Daneben sollen 2 praxisrelevante Problemfelder dargestellt und abschließend ein Ausblick in die nahe und weitere $\mathrm{Zu}$ kunft gegeben werden.

Die Ausgabe beginnt mit einer Darstellung der Therapiealgorithmen für die Colitis ulcerosa durch Kucharzik: In diesem Beitrag sind in den Behandlungsalgorithmus sowohl der Januskinaseinhibitor als auch Ustekinumab, das in Deutschland in den Markt in diesen Wochen eintritt, integriert. Analog dazu stellt der Beitrag von Fischer und Neurath die aktuellen Strategien und Algorithmen für den Morbus Crohn dar. In diesem Manuskript wird ein besonderer Schwerpunkt auf die Identifikation von Hochrisikopatienten mit der Indikation zu einer aggressiveren Therapie gelegt.

Die Verfügbarkeit einer zunehmenden Zahl und Vielfalt an Therapieoptio- nen führt manchmal dazu, dass wir die „älteren“ Standardtherapien vernachlässigen. Im Beitrag von Stange und Herrlinger werden die Daten für die Nichtbiologikatherapien praxisnah dargestellt. Neben der rein medikamentösen Therapie, die auf die Behandlung der intestinalen Entzündung abzielt, greifen die beiden folgenden Beiträge weitere zentrale Themen in der Therapie der CED auf. Dies ist zum einen der Aspekt „chronischer Schmerz und Fatigue“, der durch Sonnenberg und Biedermann mit praktischen Algorithmen für den Alltag zusammengefasst ist. Zum anderen nehmen das erhöhte Infektionsrisiko durch die immunsuppressive Therapie oder auch eine Reaktivierung von chronischen Infektionen unter Therapie zu Recht eine wichtige Stellung in unseren Therapieüberlegungen ein. Reuken, Grunert und Stallmach geben mit ihrem Beitrag eine Übersicht über alle wichtigen Aspekte, die wir in der täglichen Praxis beachten sollten.

Der abschließende Beitrag von Zeissig zeigt auf, dass die Entwicklung und Dynamik des Felds gerade erst begonnen hat: In den nächsten Jahren ist von zahlreichen weiteren Zulassungen und komplett neuen Wirkstoffklassen auszugehen. Zentral für den Einsatz dieser neuen Medikamente und ihr Wirkungssowie Nebenwirkungsprofil sind die zugrunde liegenden Wirkmechanismen. Das Verständnis dieser Mechanismen wird in Zukunft für die Betreuung dieses komplexen und heterogenen Patientenguts wesentlich sein. Wünschenswert 
wären Biomarker, die uns helfen, die initiale Strategieentscheidung für die individuell beste und erfolgversprechendste Therapie mit einer hohen Sicherheit zu treffen. Die vorhandenen Biomarker erfüllen bei kritischer Betrachtung die in sie gesetzten Erwartungen nur mit großen Einschränkungen.

Wir hoffen, dass es uns gelungen ist, mit den ausgewählten Beiträgen einen breiten Überblick über das Gebiet CED zu geben, und wünschen Ihnen viel Spaß beim Lesen dieser Ausgabe.

\section{Korrespondenzadresse}

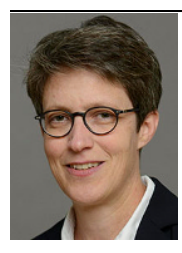

Prof. Dr. B. Siegmund

Medizinische Klinik

mit Schwerpunkt

Gastroenterologie,

Infektiologie, Rheumatologie,

Charité - Universitätsmedizin

Berlin, Campus Benjamin

Franklin

Hindenburgdamm 30

12200 Berlin, Deutschland

britta.siegmund@charite.de

Prof. Dr. med. Dr. phil.
G. Rogler
Klinik für Gastroenterologie
und Hepatologie,
UniversitätsSpital Zürich
Rämistrasse 100,8091 Zürich,
Schweiz
gerhard.rogler@usz.ch

Interessenkonflikt. B. Siegmund und G. Rogler geben an, dass kein Interessenkonflikt besteht.

\section{Leberversagen durch Angriff auf Blutgefäßzellen}

Hepatitis: Störung in der Blutversorgung als Ursache für Leberversagen

Eine Infektion mit Hepatitis-Viren kann im schlechtesten Fall zum Leberversagen führen. Ein Team der Technischen Universität München (TUM) fand nun den Grund: Immunzellen greifen Zellen des Blutgefäßsystems an und stören so die Blut- und Nährstoffversorgung des Organs. Erst das ruft die massiven Schäden hervor, die zum Leberversagen führen. Im Tiermodell wurde ein Wirkstoff identifiziert, der diese tödlichen Prozesse verhinderte.

Eine Infektion der Leber mit Hepatitis-Viren, wie dem Hepatitis-B-Virus, kann sehr unterschiedlich ablaufen: die Leberentzündung (Hepatitis) kann problemlos ausheilen, chronisch werden, sodass eine lebenslange Medikamenteneinnahme notwendig ist, oder fulminant verlaufen. Die immun-vermittelten Schäden in der Leber sind dann so stark, dass es zum Leberversagen kommt und als letzte mögliche therapeutische Maßnahme nur noch eine Lebertransplantation bleibt.

Das Ziel der Viren sind die Leberzellen (Hepatozyten). Das Immunsystem versucht, die Infektion dadurch in den Griff zu bekommen, indem sie mit Hilfe von bestimmten Immunzellen, den T-Killerzellen, die infizierten Leberzellen angreift und abtötet. Bisher wurde angenommen, dass dieser Prozess für die starken Organschäden bei einer akuten Hepatitis verantwortlich ist. Dr. Dirk Wohlleber, Forschungsgruppenleiter an der TUM, und Percy Knolle, Professor für Molekulare Immunologie, haben mit ihren Kollegen der Universitäten Würzburg und Bonn nun eine ganz andere Erklärung für das Problem gefunden: Nicht der Tod der Leberzellen, sondern Defekte im Blutgefäßsystem sind die Gründe für das Versagen des Organs.

\section{Störung der Blutversorgung durch Immunzellen}

In der Leber gibt es wichtige Zellen, die sinusoidalen Leberendothelzellen (LSECs). Sie sind die Verbindung der Leberzellen zum Blutgefäßsystem und regeln den Austausch von Nähstoffen und Sauerstoff mit dem Blut. Sie haben zudem die Fähigkeiten, kleine Teile von Viren auf ihrer Außenseite zu präsentieren, ähnlich wie Zellen des Immunsystems. Die Forscher beobachteten, dass die T-Killerzellen diese Virenstücke spezifisch erkannten. Sie hielten LSECs für infizierte Leberzellen und töteten sie. Dafür nutzten sie Proteine, die sich in die Zellmembran der Zielzelle einbauen und eine Pore bilden. Die- se sog. Perforine durchlöchern die Membran und zerstören die Zelle.

"Wir haben beobachtete, dass das Abtöten der LSECs durch die Immunzellen massive Auswirkungen auf das Lebergewebe hat. Der Blutfluss in der Leber ist massiv gestört, wodurch sehr viele - auch nicht-infizierte Leberzellen sterben. Die Auswirkung dieser Immunantwort ist sehr viel dramatischer als der Angriff auf die eigentlich infizierten Leberzellen.", erklärt Wohlleber. Möglich war diese Erkenntnis nur, weil die Wissenschaftler ein neues Mausmodell entwickelten, das ausschließlich diesen fulminanten Verlauf der Virushepatitis nachbildet.

\section{Perforin-Inhibitoren als therapeuti- sches Werkzeug}

„Erst jetzt, wo wir den eigentlichen zerstörerischen Mechanismus bei einer akuten Hepatitis kennen, können wir auch bei der Therapie in neue Richtungen denken und diesen Prozess gezielt angreifen.", so Knolle. Das Mausmodell zeigte, dass ein neuer Wirkstoff eine fulminante Hepatitis verhindert. Es handelt sich dabei um einen Perforin-Inhibitor, der die Porenbildung durch Killer-TZellen verhindert und damit auch den Angriff auf LSECs hemmte. Die Mäuse konnten durch den Einsatz des Wirkstoffs vor einer fulminanten Hepatitis bewahrt werden, weil die LSECs und damit die Blutversorgung der Leberzellen intakt blieben.

Publikation: M. Welz, et al.: Perforin inhibition protects from lethal endothelial damage during fulminant viral hepatitis, Nature Communications, 15. November 2018, DOI: 10.1038/s41467-018-07213-x(Open Access), https://www.nature.com/articles/s41467018-07213-x

Quelle: Presseaussendung 19.12.2018, Technische Universität München 